\title{
A spectroscopy facility for many
}

\author{
The 4-metre Multi-Object Spectroscopic Telescope (4MOST) is a versatile optical survey instrument that will \\ be installed on the VISTA telescope in 2022, offering medium- and high-resolution spectra and an innovative \\ operations mode, explains Principal Investigator Roelof de Jong on behalf of the 4MOST Consortium.
}

$\mathrm{T}$ he 4 MOST project is creating a new spectroscopic survey facility that will provide the highest target multiplex on the largest field-of-view in the Southern Hemisphere. The instrument is to be installed on the 4-m-class Visible and Infrared Survey Telescope for Astronomy (VISTA) of the European Southern Observatory (ESO), located at the Paranal Observatory in Chile. Expected to start its first survey at the end of 2022, it will have the capacity to obtain about 15 million spectra per year at medium and high spectral resolution in the optical wavelength range.

As the instrument name suggests, 4MOST is foreseen to address a large range of science cases. In particular its aim is to complement and enhance space missions and ground-based facilities covering large areas of the sky such as Gaia, eROSITA, Euclid, PLATO, VISTA, LSST, and SKA. The 4MOST Consortium will conduct a survey of faint Galactic stellar objects that will determine the Galactic 3D gravitational potential to unprecedented detail and measure the formation history and dynamical evolution of the Milky Way. A survey of galaxy clusters and active galactic nuclei (AGNs) discovered by the eROSITA X-ray satellite will provide very competitive constraints on cosmological parameters and dark energy evolution and determine the role of black hole and host galaxy co-evolution. Extensive galaxy, AGN, and type-Ia supernova surveys will provide strong constraints on dark energy, dark matter, and galaxy evolution.

The facility consists of a number of distributed components that will be attached to the VISTA telescope (Fig. 1). A new Wide Field Corrector will provide a $2.6^{\circ}$ field of view. At the focal surface of $535-\mathrm{mm}$ diameter, a fibre positioner system based on the tilting spine principle moves 2,436 glass fibres to astronomical targets of interest with an accuracy of $5 \mu \mathrm{m}$. The fibres transport the target's light to two medium-resolution spectrographs $(R=\lambda / \Delta \lambda \approx 5,000)$ with a wavelength coverage of $370-950 \mathrm{~nm}$ and to one high-resolution spectrograph
$(R \approx 20,000)$ covering three optical wavelength bands. Each spectrograph has three fixed-wavelength channels equipped with high quantum efficiency CCD detectors with $6,144 \times 6,160$ pixels.

Running as a dedicated survey facility at Paranal Observatory with its many clear nights and high image quality, the productivity of 4MOST is predicted to be very good. The instrument system consists of state-of-the-art components for high throughput and in a total exposure time of two hours is expected to deliver highresolution spectra with a signal-to-noise ratio $S / N=100 \AA^{-1}$ for objects with a flux of $m_{\mathrm{AB}}=16$ mag, yielding high-quality stellar elemental abundances. In the same exposure time, the medium-resolution spectrographs reach $\mathrm{S} / \mathrm{N}=30 \AA^{-1}$ for $m_{\mathrm{AB}}=18$ mag objects to obtain good stellar parameters and $\mathrm{S} / \mathrm{N}=3 \AA^{-1}$ for $m_{\mathrm{AB}}=22$ mag objects, sufficient to measure the redshifts of galaxies and AGNs.

An innovative operations mode is foreseen for 4MOST. Normally one science project is scheduled at a time on a telescope, but 4MOST has such a high multiplex that many programmes will not have enough targets to fill all fibres, and hence 4MOST will observe many science cases simultaneously. This also allows science cases that have sparse targets spread over the entire sky to be mixed in with other projects that have sufficient target densities to fill most fibres. ESO will issue a call for Letters of Intent in the fourth quarter of 2019 to solicit proposals from its astronomical community, to be mixed in with the programme that will be carried out by the 4MOST consortium. All 4MOST data and derived analysis products will be distributed to the entire astronomical community.

The 4MOST subsystems are currently in construction with full system integration starting at the Leibniz Institute for Astrophysics in Potsdam at the end of 2019. Full integration and testing in Europe is expected to be completed by early 2022 , after which the instrument will be shipped to Paranal Observatory to be integrated and commissioned at the telescope. Science

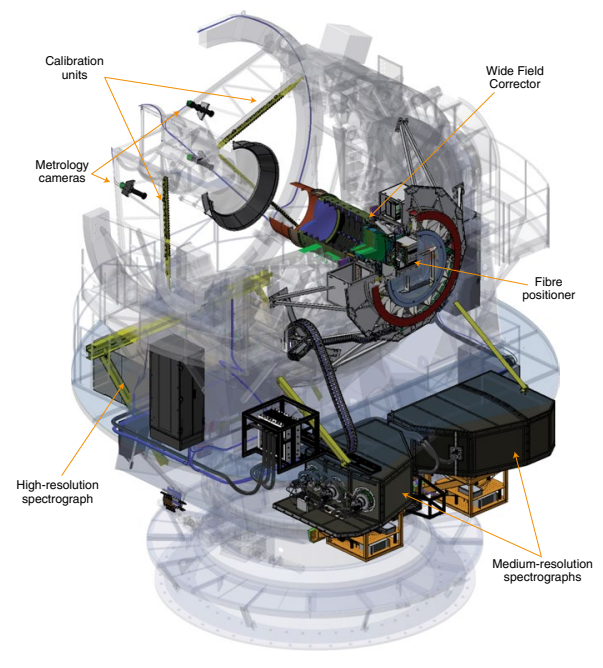

Fig. 1 | Schematic of the 4MOST components integrated with VISTA.

verification is expected to be completed at the end of 2022, after which 4MOST will start its first five-year survey.

$4 \mathrm{MOST}$ is being developed by a team of more than 350 scientists, engineers, managers, and support staff. Consortium partners are Leibniz-Institut für Astrophysik Potsdam (AIP; lead institute), Australian Astronomical Optics - Macquarie, Centre de Recherche Astrophysique de Lyon, European Southern Observatory, Institute of Astronomy - Cambridge, Max-Planck-Institut für Astronomie, MaxPlanck-Institut für extraterrestrische Physik, NOVA/ASTRON Dwingeloo, Zentrum für Astronomie der Universität Heidelberg, École polytechnique fédérale de Lausanne, Lunds Universitet, Rijksuniversiteit Groningen, Universität Hamburg, University of Western Australia, and Uppsala Universitet.

Roelof S. de Jong (D)

Leibniz-Institut für Astrophysik Potsdam (AIP),

Potsdam, Germany.

e-mail:rdejong@aip.de

Published online: 7 June 2019

https://doi.org/10.1038/s41550-019-0808-X 